\title{
Nonexistence of Coherent Structures in Two-dimensional Inviscid Channel Flow
}

\begin{abstract}
H. Kalisch*
University of Bergen, Department of Mathematics, P.O. Box 7800, 5020 Bergen, Norway

Abstract. Two-dimensional inviscid channel flow of an incompressible fluid is considered. It is shown that if the flow is steady and features no horizontal stagnation, then the flow must necessarily be a parallel shear flow.
\end{abstract}

Keywords and phrases: inviscid channel flow, hodograph transform, maximum principle, steady flow

Mathematics Subject Classification: 35J15, 35Q31, 76E05

\section{Introduction}

We consider the problem of steady two-dimensional channel flow. This flow is an important test case for stability theories, and there is a vast body of work concerning the stability of parallel shear flows both in the viscous and inviscid case [5]. For instance, in the inviscid case, one may find conditions on the stability of the flow with respect to inflection points of the horizontal velocity profile. Theses studies also include the investigation of critical layer flows [5] which feature the famous cat's eye [20], and also appear in the study of surface waves [24]. One may also consider channel flows under the influence of the Coriolis force [16]. There have also been studies of the stability of non-parallel steady flows, such as in [11] for the case of bounded domains and periodic flows.

In the viscous case, the study of instability of steady flows is also an active field of research $[13,23,26]$. The channel flow is one of the most important examples in the study of transition to turbulence [22], and the recent discovery of unstable traveling waves in viscous channel and pipe flow has given new impetus to the study of transition from laminar flow to turbulence [25].

It follows from the result of this note that there cannot be any steady translational structures in twodimensional inviscid channel flow, unless the flow contains points where the horizontal component of the particle velocity is equal to the phase speed of the steady profile.

Imagine a fluid contained in a horizontal channel of uniform width and depth, and with an idealized infinite expanse in the principal horizontal direction. Suppose the fluid is incompressible and inviscid, and the flow is two-dimensional in the sense that the variation of the flow in the direction transverse to the direction of propagation is negligible. One possible configuration is that the flow is a parallel shear flow, in other words, the velocity is directed exclusively in the horizontal direction. On the other hand

\footnotetext{
*Corresponding author. E-mail: henrik.kalisch@math.uib.no
} 


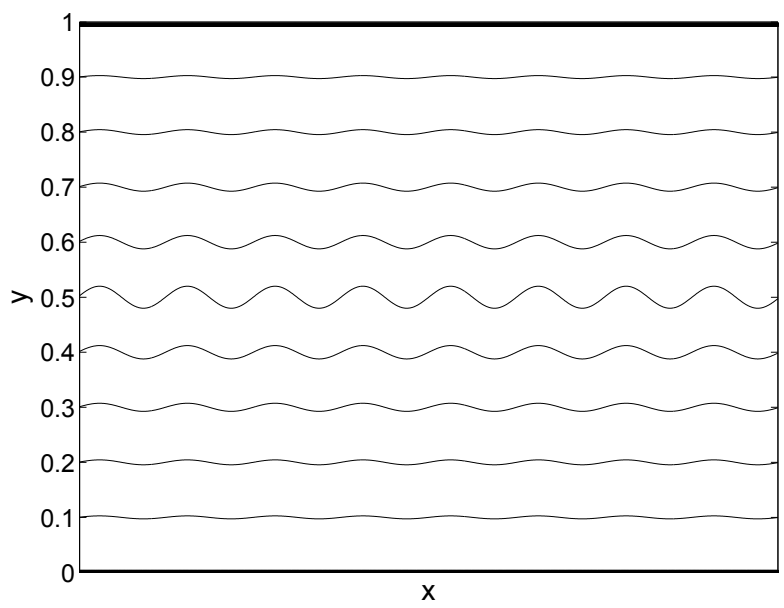

FIGURE 1. A smooth periodic streamline pattern. In this case, the height of the channel is $H=1$. It follows from Theorem 1.1 that this kind of steady flow cannot exist in an inviscid fluid.

one might imagine other types of coherent structures, such as the case where the flow is steady, and the streamlines are periodic in the direction of propagation (cf. Figure 1). The main result of this note is the proof that such structures do not exist unless the horizontal particle velocity becomes equal to the propagation velocity of the profile. At such points, the internal structure starts to break, and this can be a starting point for the creation of turbulence.

The result may be rephrased by saying that, in the absence of viscosity and internal breaking, the only possible two-dimensional steady flow is the parallel shear flow. To prove this result, we bring techniques to bear which have been previously used mainly for free-surface problems $[2-4,9,18,19]$. In particular, we use a hodograph transform to put the equations in a convenient form where a maximum principle can be applied.

Before the theorem is formalized, let us recall the equations for two-dimensional channel flow. As the fluid is assumed to be inviscid, the governing equations are the Euler equations, supplemented with appropriate boundary conditions. Let $x$ denote the horizontal, and $y$ the vertical variable. We assume that there is no variation of the flow in the transverse direction, so that the flow is two-dimensional. The coordinates are chosen such that the top of the channel is at height $y=H$, and the bottom is at $y=0$. Let $u(x, y, t)$ and $v(x, y, t)$ be the horizontal and vertical velocity of the fluid, respectively. The Euler momentum equations are

$$
\left.\begin{array}{rl}
u_{t}+u u_{x}+v u_{y} & =-p_{x} \\
v_{t}+u v_{x}+v v_{y} & =-p_{y}
\end{array}\right\}
$$

where $p(x, y, t)$ is the dynamic pressure. For convenience, the density of the fluid is assumed to be unity. Note that since no free surface is involved, gravity plays no role in this problem. The incompressibility is expressed by the condition that

$$
u_{x}+v_{y}=0
$$

At the top and bottom, the fact that the fluid cannot flow through the boundary is expressed by the condition

$$
v=0 \quad \text { on } \quad y=H,
$$

and

$$
v=0 \quad \text { on } \quad y=0 .
$$


Now assume that the solution is given by a steady profile. By a steady profile, we mean a profile which propagates to the right or to the left at a fixed phase speed $c$ without changing its shape over time. For such steady solutions, the velocity $(u, v)$, and the pressure $P$ depend only on the quantity $x-c t$, rather than on $x$ and $t$ separately. Thus we may write the dependent variables in the form $u(x, y, t)=U(x-c t, y)+c$, $v(x, y, t)=V(x-c t, y)$, and $p(x, y, t)=P(x-c t, y)$. The momentum equations are then

$$
\left.\begin{array}{r}
U U_{x}+V U_{y}=-P_{x} \\
U V_{x}+V V_{y}=-P_{y}
\end{array}\right\}
$$

and the incompressibility and boundary conditions have not changed. Now it is evident that a parallel shear flow $U(x, y)=U_{0}(y), V(x, y)=0$ with constant pressure is a solution of (1.5). The question then arises whether there are steady flows other than parallel shear flows. In this paper, it is shown that if there are no points, where the internal structure start to break, i.e. where $U=0$, then the only possible steady flow is a parallel shear flow. In the original reference frame (i.e. equations (1.1)), this condition requires that the horizontal component of the particle velocity be less than the phase velocity of the steady profile, i.e. $|u|<|c|$. In light of these considerations, the main result of this paper may be formulated as follows.

Theorem 1.1. Let $U, V$ and $P$ describe a steady solution of (1.5), (1.2), (1.3) and (1.4) with $U>0$. Then $V \equiv 0$.

In the next section, the problem will be formulated in terms of the stream function. Some preliminary observations will be made, and the crucial maximum principle to be used in the proof of Theorem 1.1 will be recalled. Then in Section 3, the proof of Theorem 1.1 will be given.

\section{Preliminaries}

Assume that a solution such as described in Theorem 1.1 exists. The incompressibility guarantees the existence of the stream function $\psi$ which is defined by requiring that

$$
\psi_{x}=-V \quad \text { and } \quad \psi_{y}=U=u-c .
$$

We may normalize the stream function to be 0 on the boundary $y=0$. Defining the mass flux (in the traveling frame) across a vertical line $(x, y)=\left(x_{0}, y\right)$ in the channel by

$$
Q_{m}=\int_{0}^{H} U\left(x_{0}, y\right) d y
$$

it appears that then $\psi$ takes the value $Q_{m}$ on the upper boundary $y=H$. To normalize the problem we assume that the profile is traveling in the direction of decreasing $x$-values (to the left) in the inertial frame, so that $c<0$. In that case, $Q_{m}$ which is defined in the moving reference frame is positive since the absence of internal breaking implies $|u|<|c|$, so that $U=u-c>0$. The definition of the stream function shows that

$$
-\triangle \psi=\omega,
$$

where $\omega(x, y)=V_{x}-U_{y}$ is the vorticity of the flow at a point $(x, y)$ in the fluid. Now it can be shown that if $U>0$, then the vorticity is constant on any streamline, so that it is given by

$$
\omega=\gamma(\psi),
$$

for some function $\gamma:\left[0, Q_{m}\right] \rightarrow \mathbb{R}$. For the proof of this fact, note that it can be shown from the momentum equations that locally, the gradients of $\omega$ and $\psi$ are collinear. For the proof that this relation extends to a global one, see [3]. 
The problem (1.2), (1.3) and (1.4) may now be formulated in terms of the stream function $\psi$ as follows. The equation for the stream function is

$$
\triangle \psi=-\gamma(\psi) \text { on } \quad 0<y<\eta(x),
$$

and the boundary conditions are

$$
\left.\begin{array}{lll}
\psi=Q_{m} & \text { on } & y=H \\
\psi=0 & \text { on } & y=0
\end{array}\right\}
$$

The momentum equations (1.5) have been used to show the existence of the vorticity function $\gamma$. Now in order to prove that $V \equiv 0$, it is tempting to differentiate equation (2.3) to find an elliptic problem for $V=-\psi_{x}$. Such an approach has been used successfully in [8], but it does not appear to work in the present case, because there are no natural conditions to be imposed on the derivative $\gamma^{\prime}(\psi)$. However, as we will see in the next section, the problem can be transformed into a different form in which it is amenable to maximum principle methods.

The version of the strong maximum principle for elliptic partial differential operators to be used here can be found on page 35 in [12]. Suppose we have an elliptic operator of the form

$\mathcal{L}=A^{11}(x, y) \partial_{x x}+\left\{A^{12}(x, y)+A^{21}(x, y)\right\} \partial_{x y}+A^{22}(x, y) \partial_{y y}+B^{1}(x, y) \partial_{x}+B^{2}(x, y) \partial_{y}+C(x, y)$,

where $A^{12}=A^{21}$.

Theorem 2.1. Let $\mathcal{L}$ be locally uniformly elliptic in the domain $\Omega$, and let $\lambda(x, y)$ be the minimum eigenvalue of the symmetric $2 \times 2$-matrix with the components $A^{i j}(x, y)$. Let $C(x, y) \leq 0$, and suppose that $C / \lambda$ is locally bounded. Suppose the function $w$ is twice continuously differentiable and satisfies $\mathcal{L} w=0$. If $w$ achieves a non-negative maximum or a non-positive minimum in the interior of the domain $\Omega$, then $w$ is constant on $\Omega$.

This theorem is standard in the theory of elliptic equations. However, one important feature of this maximum principle is that it holds on unbounded domains.

\section{Proof of Theorem 1.1}

The proof of Theorem 1.1 is based on further equivalent formulation of the problem based on a type of hodograph transformation. We introduce the new independent variables $\chi=x$ and $\xi=\psi$, and the new dependent variable $Y(\chi, \xi)=y$. This transformation was introduced by Dubreil-Jacotin [6], but we use it in a way which is similar to the work presented in [3]. However, since the variables are normalized somewhat differently, different notation than in [3] is used. Note that the transformation is a one-to-one change of variables so long as $\psi_{y}>0$, which is guaranteed by the assumption that $U>0$. Furthermore, in [3] it was proved that the relations

$$
Y_{\chi}=\frac{V}{U}, \quad Y_{\xi}=\frac{1}{U}
$$

and

$$
U=\frac{1}{Y_{\xi}}, \quad V=\frac{Y_{\chi}}{Y_{\xi}}
$$

hold. Moreover, it is immediate that the derivatives transform in the following way:

$$
\partial_{x}=\partial_{\chi}-\frac{Y_{\chi}}{Y_{\xi}} \partial_{\xi}, \quad \partial_{y}=\frac{1}{Y_{\xi}} \partial_{\xi}
$$

Now, as in $[3,9]$, it can be verified that the equation (2.3) transforms into the following equation for $Y$ :

$$
\left(1+Y_{\chi}^{2}\right) Y_{\xi \xi}-2 Y_{\chi} Y_{\xi} Y_{\chi \xi}+Y_{\xi}^{2} Y_{\chi \chi}-\gamma(\xi) Y_{\xi}^{3}=0
$$


The domain of $Y$ is the infinite strip $\Omega=\left\{(\chi, \xi) \in \mathbb{R}^{2} \mid 0<\xi<Q_{m}\right\}$, and the boundary conditions are given by $Y(\chi, 0)=0$ and $Y\left(\chi, Q_{m}\right)=H$.

Recall that our aim is to show that $V \equiv 0$. Contemplating the relation $Y_{\chi}=\frac{V}{U}=\frac{v}{u-c}$ and the assumption that $u-c>0$, it is plain that this goal will be accomplished if it can be shown that $Y_{\chi} \equiv 0$. To find an equation for $Y_{\chi}$, we differentiate (3.1) with respect to $\chi$ to obtain

$$
\begin{aligned}
2 Y_{\chi} Y_{\chi \chi} Y_{\xi \xi}+Y_{\xi \xi \chi}+Y_{\chi}^{2} Y_{\xi \xi \chi}-2 Y_{\chi \chi} Y_{\xi} Y_{\chi \xi}-2 Y_{\chi} Y_{\xi \chi} Y_{\chi \xi}-2 Y_{\chi} Y_{\xi} Y_{\xi \chi \chi} & \\
& +2 Y_{\xi} Y_{\xi \chi} Y_{\chi \chi}+Y_{\xi}^{2} Y_{\chi \chi \chi}-3 \gamma(\xi) Y_{\xi}^{2} Y_{\xi \chi}=0
\end{aligned}
$$

This reduces to the expression

$$
2 Y_{\chi} Y_{\chi \chi} Y_{\xi \xi}+Y_{\xi \xi \chi}+Y_{\chi}^{2} Y_{\xi \xi \chi}-2 Y_{\chi} Y_{\chi \xi}^{2}-2 Y_{\xi} Y_{\chi} Y_{\xi \chi \chi}+Y_{\xi}^{2} Y_{\chi \chi \chi}-3 \gamma(\xi) Y_{\xi}^{2} Y_{\xi \chi}=0
$$

Collecting the second, third, fifth and sixth terms in one bracket, and defining $w=Y_{\chi}$, we gain the equation

$$
\left\{w_{\xi \xi}+Y_{\chi}^{2} w_{\xi \xi}-2 Y_{\xi} Y_{\chi} w_{\xi \chi}+Y_{\xi}^{2} w_{\chi \chi}\right\}+\left\{2 Y_{\chi} Y_{\xi \xi} w_{\chi}-3 \gamma(\xi) Y_{\xi}^{2} w_{\xi}\right\}-2 Y_{\xi \chi}^{2} w=0 .
$$

Thus $w$ satisfies the equation $\mathcal{L} w=0$, where the operator $\mathcal{L}$ is defined by

$$
\mathcal{L}=\left\{\left(1+Y_{\chi}^{2}\right) \partial_{\xi \xi}-2 Y_{\xi} Y_{\chi} \partial_{\xi \chi}+Y_{\xi}^{2} \partial_{\chi \chi}\right\}+\left\{2 Y_{\xi} Y_{\xi \chi} \partial_{\chi}-3 \gamma(\xi) Y_{\xi}^{2} \partial_{\xi}\right\}-2 Y_{\xi \chi}^{2} .
$$

This operator is elliptic, as can be seen by observing that the matrix

$$
A=\left(\begin{array}{cc}
1+Y_{\chi}^{2} & -Y_{\chi} Y_{\xi} \\
-Y_{\chi} Y_{\xi} & Y_{\xi}^{2}
\end{array}\right)
$$

is positive-definite. Indeed we have $1+Y_{\chi}^{2}>0$ and $\operatorname{det}(A)=Y_{\xi}^{2}>0$ since $Y_{\xi}=\frac{1}{U}=\frac{1}{u-c}>0$. Thus it follows that $\mathcal{L}$ is locally uniformly elliptic. The first-order terms have the coefficients $B_{1}=2 Y_{\chi} Y_{\chi \xi}$ and $B_{2}=-3 \gamma(\xi) Y_{\chi}^{2}$ whose signs do not play a role. Finally, note that

$$
C(\chi, \xi)=-2 Y_{\xi \chi}^{2} \leq 0,
$$

and if $\lambda(\chi, \xi)$ denotes the lowest eigenvalue of the matrix $A$ at a point $(\chi, \xi)$, then the function $C / \lambda$ is locally bounded (on any compact set). From the boundary condition $Y\left(\chi, Q_{m}\right)=H$, we obtain $w=Y_{\chi}=0$ on $\xi=Q_{m}$. From $Y(\chi, 0)=0$, it follows that $w=Y_{\chi}=0$ on $\xi=0$.

Now the strong maximum principle for locally uniformly elliptic operators with $C \leq 0$ as stated in Theorem 2.1 implies that if $\mathcal{L} w=0$, then the function $w$ cannot assume a non-negative maximum or non-positive minimum in the interior unless it is constant. Since $w$ is identically 0 on the top and bottom of the strip $\Omega$ it cannot take any other value in the interior. Thus $w \equiv 0$. Since $V=\frac{Y_{\xi}}{Y_{\chi}}=\frac{w}{U}$, and $U>0$, we conclude that $V \equiv 0$, and the flow is a parallel shear flow.

\section{Conclusion}

Theorem 1.1 shows that there are no steady translatory streamline patterns in two-dimensional inviscid channel flow unless the flow also features points where $U=0$, which corresponds to an internal fluid structure which is starting to break.

On the other hand, smooth coherent structures in the channel flow might exist in the presence of molecular viscosity. However, the balance is then most likely between nonlinearity and viscous dissipation at the boundaries.

It is interesting to note that the breaking criterion $U=0$ can also be applied to model equations for waves at a free surface, such as the Boussinesq system. In this case, a limiting amplitude for the existence of traveling waves can be found [1]. Note also that a time-dependent analysis of a singular profile in the 
viscous case has been provided in [10]. Moreover, one may also study the effects of bottom topography and three-dimensional effects [17].

Still another possibility allowing the existence of smooth coherent structures is if the density of the fluid is not homogeneous. In this case, gravity cannot be eliminated from the problem, and the restoring force is due to buoyancy. Indeed, such a configuration has been investigated in [7], and in many other works since.

Acknowledgements. The author would like to thank Mats Ehrnström for valuable discussions. The work in this article was partially supported by the Research Council of Norway.

\section{References}

[1] M. Bjørkavåg, H. Kalisch. Wave breaking in Boussinesq models for undular bores. Phys. Lett. A, 375 (2011), $157-1578$.

[2] A. Constantin, M. Ehrnström, E. Wahlén. Symmetry of steady periodic gravity water waves with vorticity. Duke Math. J., 140 (2007), 591-603.

[3] A. Constantin, W. Strauss. Exact steady periodic water waves with vorticity. Comm. Pure Appl. Math., 57 (2004), $481-527$.

[4] W. Craig. Non-existence of solitary water waves in three dimensions. Philos. Trans. R. Soc. Lond. Ser. A Math. Phys. Eng. Sci., 360 (2002), 2127-2135.

[5] P.G. Drazin, W.H. Reid. Hydrodynamic stability. Cambridge University Press, Cambridge, 2004.

[6] M.-L. Dubreil-Jacotin. Sur la détermination rigoureuse des ondes permanentes périodiques d'ampleur finie. J. Math. Pures Appl., 13 (1934), 217-291.

[7] M.-L. Dubreil-Jacotin. Sur les théorèmes d'existence relatifs aux ondes permanentes périodiques 'a deux dimensions dans les liquides hétérogènes. J. Math. Pures Appl., 16 (1937), 43-67.

[8] M. Ehrnström. A note on surface profiles for symmetric gravity waves with vorticity. J. Nonlinear Math. Phys., 13 (2006), 1-8.

[9] M. Ehrnström. Uniqueness for steady periodic water waves with vorticity. Int. Math. Res. Not., 2005 (2005), $3721-3726$.

[10] L.E. Fraenkel. On Kelvin-Stuart vortices in a viscous fluid. Philos. Trans. R. Soc. Lond. Ser. A Math. Phys. Eng. Sci., 366 (2008), 2717-2728.

[11] S. Friedlander, W. Strauss, M. Vishik. Nonlinear instability in an ideal fluid. Ann. Inst. H. Poincaré Anal. Non Linéaire, 14 (1997), 187-209.

[12] D. Gilbarg, N.S. Trudinger. Elliptic partial differential equations of second order. Grundlehren der Mathematischen Wissenschaften 224, Springer, Berlin-New York, 1977.

[13] O. Goubet. A relation between the pressure gradient and the flux for the general channel flow problem. Appl. Math. Optim., 34 (1996), 361-365.

[14] B. Hof, C.W.H. van Doorne, J. Westerweel, F.T.M. Nieuwstadt, H. Faisst, B. Eckhardt, H. Wedin, R.R. Kerswell, F. Waleffe. Experimental Observation of Nonlinear Traveling Waves in Turbulent Pipe Flow. Science, 10 (2004), 15941598.

[15] V.M Hur, Z. Lin. Unstable surface waves in running water. Comm. Math. Phys., 282 (2008), 733-796.

[16] N.H. Ibragimov, R Aitbayev, R.N. Ibragimov. Three-dimensional nonlinear rotating surface waves in channels of variable depth in the presence of formation of a small perturbation of atmospheric pressure across the channel. Commun. Nonlinear Sci. and Numer. Simul., 14 (2009), 3811-3820.

[17] R.N. Ibragimov, D.E. Pelinovsky. Three-dimensional gravity waves in a channel of variable depth. Commun. Nonlinear Sci. and Numer. Simul., 13 (2008), 2104-2113.

[18] H. Kalisch. Periodic traveling water waves with isobaric streamlines. J. Nonlinear Math. Phys., 11 (2004), $461-471$.

[19] H. Kalisch. A uniqueness result for periodic traveling waves in water of finite depth. Nonlinear Anal., 58 (2004), $779-785$.

[20] W. Thomson. On disturbing infinity in Lord Rayleigh's solution for waves in a plane vortex stratum. Nature, 23 (1880), 45-46.

[21] H. Lamb. Hydrodynamics. Cambridge University Press, London, 1924.

[22] P. Moin, J. Kim. Numerical investigation of turbulent channel flow. J. Fluid Mech., 118 (1982), $341-377$.

[23] A.E. Trefethen, L.N. Trefethen, P.J. Schmid. Spectra and pseudospectra for pipe Poiseuille flow. Comput. Methods Appl. Mech. Engrg., 175 (1999), 413-420.

[24] E. Wahlén. Steady water waves with a critical layer. J. Differential Equations, 246 (2009), 2468-2483.

[25] F. Waleffe. Homotopy of exact coherent structures in plane shear flows. Phys. Fluids, 15 (2003), $1517-1534$.

[26] P.O. Åsén, G. Kreiss. On a rigorous resolvent estimate for plane Couette flow. J. Math. Fluid Mech., 9 (2007), 153-180. 